\title{
Age at menarche and the risk of diabetic microvascular complications in patients with type 1 diabetes
}

\author{
Valma Harjutsalo ${ }^{1,2,3,4}$. Christine Maric-Bilkan ${ }^{5}$ - Carol Forsblom ${ }^{1,2,3}$ • \\ Per-Henrik Groop ${ }^{1,2,3,6}$ o on behalf of the FinnDiane Study Group
}

Received: 6 August 2015 / Accepted: 2 November 2015 / Published online: 25 November 2015

(C) Springer-Verlag Berlin Heidelberg 2015

\begin{abstract}
Aims/hypothesis The aim of this study was to evaluate the relationship among age at onset of diabetes, age at onset of menarche and risk of diabetic nephropathy and laser-treated retinopathy in type 1 diabetes.

Methods Data related to age at menarche were collected through questionnaires and were available for 1,304 women who participated in the Finnish Diabetic Nephropathy Study (FinnDiane). A possible association between age at menarche and diabetic nephropathy and retinopathy was investigated. Results There was an inverse relationship between the age at onset of diabetes and age at menarche: the younger the age at onset of diabetes, the higher the age at menarche $(p<0.0001)$. A non-linear relationship between the age of menarche and
\end{abstract}

Electronic supplementary material The online version of this article (doi:10.1007/s00125-015-3816-0) contains peer-reviewed but unedited supplementary material, which is available to authorised users.

Per-Henrik Groop

per-henrik.groop@helsinki.fi

1 Folkhälsan Research Center, Biomedicum Helsinki, University of Helsinki, PO Box 63, 00014 Helsinki, Finland

2 Abdominal Center Nephrology, University of Helsinki and Helsinki University Hospital, Helsinki, Finland

3 Research Program Unit, Diabetes and Obesity, University of Helsinki, Helsinki, Finland

4 The Chronic Disease Prevention Unit, National Institute for Health and Welfare, Helsinki, Finland

5 Vascular Biology and Hypertension Branch, Division of Cardiovascular Sciences, National Institute of Heart, Lung and Blood, National Institutes of Health, Bethesda, MD, USA

6 Baker IDI Heart and Diabetes Institute, Melbourne, Victoria, Australia risk of diabetic microvascular complications was found in patients with diabetes onset before menarche, but there was no such association in patients with diabetes onset after menarche. Women with delayed menarche ( $>$ mean age +2 years) had a $2.30(95 \%$ CI 1.27, 4.17; $p<0.006)$ times higher risk of nephropathy compared with the women who underwent menarche at the mean age \pm 2 years. Delayed menarche also increased the risk of retinopathy (OR 2.34 [95\% CI 1.36, 4.01]). After excluding patients with nephropathy, the OR for retinopathy was 2.11 (95\% CI 1.15, 3.90). Earlier menarche $(<$ mean age -2 years $)$ did not have any effect on this risk. Conclusions/interpretation Delayed menarche was associated with an increased risk of diabetic nephropathy and retinopathy, whereas early menarche was not. Delayed menarche may be used as a new tool to identify women at risk of diabetic microvascular complications.

Keywords Age at menarche - Age at onset of diabetes . Delayed menarche $\cdot$ Diabetic nephropathy $\cdot$ Diabetic retinopathy $\cdot$ Puberty $\cdot$ Type 1 diabetes

\begin{tabular}{ll}
\multicolumn{2}{l}{ Abbreviations } \\
ESRD & End-stage renal disease \\
FinnDiane & Finnish Diabetic Nephropathy Study \\
GAM & Generalised additive modelling \\
HPG & Hypothalamus-pituitary-gonadal \\
SBP & Systolic BP
\end{tabular}

\section{Introduction}

Previous studies have shown that age at onset of type 1 diabetes has an effect on the risk of microvascular complications, including diabetic nephropathy $[1,2]$ and diabetic 
proliferative retinopathy [3]. Diabetic end-organ complications rarely occur before puberty, and the onset of puberty accelerates microvascular complications [4-7]. These observations suggest an association between sex hormones and diabetic end-organ complications. Providing further support for a close relationship between the development of diabetic complications and sex hormones, especially in women with type 1 diabetes, is the fact that these women commonly experience reproductive health issues including delayed menarche and puberty, an increased rate of menstrual irregularities, hyperandrogenism, a reduced ability to conceive, a high risk of adverse pregnancy outcomes (including spontaneous abortions, stillbirths and congenital anomalies), and premature menopause [8-12]. What remains unclear is whether any of these complications related to the reproductive system can contribute to the risk of other, non-reproductive organ complications associated with diabetes, such as diabetic nephropathy and diabetic retinopathy. Women with type 1 diabetes exhibit a much higher incidence of renal disease compared with nondiabetic women [13]. One of the factors contributing to this observation may be the abnormal regulation of sex hormone concentrations associated with diabetes. Indeed, both clinical and experimental data suggest that type 1 diabetes may be associated with reduced circulating estradiol levels [14-16]. These observations, coupled with the fact that puberty accelerates renal dysfunction, support the notion of a correlation between reproductive and renal abnormalities in type 1 diabetes.

Several studies have reported delayed menstruation in young women with type 1 diabetes [17-19]. While some studies concluded that delayed menarche is related to poor glycaemic control, other studies have reported that menarche in type 1 diabetes is still delayed when there is good metabolic control [20-22]. In contrast, some studies show no association between the age at menarche and diabetes [23, 24], suggesting that there is still no clear understanding of whether or not the presence of diabetes affects the onset of menstruation and whether this relationship might be dependent on the age at onset of type 1 diabetes. Interestingly, over the years, the age of menarche has progressively been declining and this secular trend has also been observed in girls both with and without type 1 diabetes throughout the world [25].

Thus, since studies suggest that type 1 diabetes may affect the age at menarche and that both the age at onset of type 1 diabetes and pubertal changes affect the risk of diabetic microvascular complications, we hypothesised that age at onset of menarche may be an important determinant of the risk of diabetic nephropathy and retinopathy in type 1 diabetes. Therefore, the aim of this study was to evaluate the effect of the combined type 1 diabetes-puberty-driven cascade on the risk of diabetic microvascular complications. We aimed to first, evaluate the effect of age at onset of diabetes on the timing of menarche in a large continuum of ages at onset of diabetes and, second, evaluate the risk of microvascular complications after dividing the study population into groups with diabetes onset before and after the onset of menarche.

\section{Methods}

Data used for the analyses were derived from the Finnish Diabetic Nephropathy Study (FinnDiane). FinnDiane is a nationwide, comprehensive multicentre study with the aim of identifying genetic and environmental risk factors for diabetic complications, with a special emphasis on diabetic nephropathy in patients with type 1 diabetes. A detailed description of the FinnDiane recruitment protocol was previously reported [24]. Briefly, all adult (age $\geq 18$ years) patients with type 1 diabetes from 21 university and central hospitals, 33 district hospitals, and 26 primary health-care centres across Finland were asked to participate; the participation rate was $78 \%$. The age range of patients at the baseline visit was 18-76 years. Type 1 diabetes was defined by an age at onset of diabetes of $<40$ years with insulin treatment initiation within 1 year of diagnosis.

At the baseline visit between 1994 and 2011, study patients underwent a throughout clinical examination at a regular visit to their attending physician at each FinnDiane centre. During this visit, their BP was measured and blood samples collected for lipid, $\mathrm{HbA}_{1 \mathrm{c}}$ and other measurements. Data regarding the presence of microalbuminuria, macroalbuminuria or endstage renal disease (ESRD), as well as laser photocoagulation of eyes, were obtained from the medical files by the attending physician using a standardised questionnaire. In the present study, the diagnosis of macroalbuminuria or ESRD was defined as diabetic nephropathy and laser photocoagulation as severe retinopathy.

Data related to reproductive health history and related health issues were collected in the year 2011 and onwards through a questionnaire obtained from 1,325 women. The self-administered study questionnaire included questions about reproductive health including age at menarche. Of these patients, 21 could not provide information on the age of menarche, leaving 1,304 eligible study participants. These patients were divided according to the decade of their birth; 1944 or before, 1945-1954, 1955-1964, 1965-1974, 1975-1984 and 1985 or after; deviation in the age at menarche from the group mean was determined for each birth decade. The association between deviation from the group mean and risk of diabetic nephropathy and severe retinopathy was studied.

Statistical analysis The relationship between age at menarche and age at onset of diabetes was first studied using the generalised additive modelling (GAM) without a priori assumptions about type of relationship. Similarly, the relationship between 
age at menarche and risk of diabetic nephropathy and severe retinopathy was studied using GAM. Because data on the diagnosis of nephropathy or retinopathy were not available for all patients, the binary response variable of whether the patients had diabetic nephropathy or severe retinopathy at the baseline was used. GAM was thus conducted under binomial distribution and the link function was logit. GAM is an extension of the generalised linear model that allows the inclusion of non-parametric smoothing functions to identify potential non-linearity in the relationship between the independent and the dependent variables [26, 27]. The generalised crossvalidation function was used as a criterion for the selection of the smoothing parameters to determine an appropriate level of smoothing. The $d f$ values indicating the smoothing level were $4.14,3.37$ and 1.16 for diabetic nephropathy, retinopathy, and retinopathy when patients with nephropathy were excluded, respectively. Partial residual plots are provided to show the relationship between age at menarche and the risk of microvascular complications.

Logistic regression analyses were performed to quantify the effect of delayed menarche on the risk of diabetic microvascular complications. Based on the results drawn from GAM, data were divided into three groups relative to the mean age at menarche: mean age \pm 2 years (reference group); less than the mean age -2 years; and more than the mean age+ 2 years. Multivariable analyses were conducted by adjusting for the following well-defined key risk factors: age at onset of diabetes; duration of diabetes; $\mathrm{BMI}$; smoking status; $\mathrm{HbA}_{1 \mathrm{c}}$; systolic BP (SBP); HDL-cholesterol and triacylglycerol levels. $\mathrm{HbA}_{1 \mathrm{c}}$ was measured once at the baseline evaluation; therefore, the effect of metabolic control on age of menarche could not to be taken into account. All analyses were performed using SAS statistical software (version 9.3, SAS Institute, Cary, NC, USA) and R open source software (www.r-project.org). GAM models were fitted using the mgcv library in R [28].

The ethics committees of all participating centres approved the study protocol. Written informed consent was obtained from each patient and the study was performed in accordance with the Declaration of Helsinki as revised in the year 2000 .

\section{Results}

Information on 1,304 women was available for inclusion in the analyses. The age range at the time of questionnaire collection was $18-85$ years (mean age $47.7 \pm 11.5$ ). Of the 1,304 participants, 600 had diabetes onset before menarche (premenarcheal diabetes) and 704 after (post-menarcheal diabetes). A total of 117 (19.5\%) patients in the pre-menarcheal diabetes group and $50(7.1 \%)$ patients in the postmenarcheal diabetes groups were diagnosed with diabetic nephropathy at the baseline visit. The corresponding numbers were $236(39.3 \%)$ and 96 (13.6\%), respectively, for severe retinopathy.

The age at menarche varied from 9 to 18 years. The clinical characteristics of patients grouped by pre- and postmenarcheal diabetes onset are shown in Table 1. Premenarcheal diabetes was associated with a shorter stature, worse glycaemic control and blood lipid profiles, and a higher prevalence of antihypertensive medication. Table 2 shows the clinical characteristics of patients with pre-menarcheal type 1 diabetes according to the age at menarche. Pre-menarcheal diabetes shifts the distribution towards an older age at menarche and slightly distorts the normal distribution of age at menarche. Patients with a delayed menarche had the worst SBP, but the difference in SBP was no longer significant after adjustment for age and duration of diabetes. However, the frequency of antihypertensive medication remained significant after adjustment. Early and delayed menarche were both associated with a shorter stature.

The mean $( \pm \mathrm{SD})$ age at menarche was higher in women with pre-menarcheal diabetes ( $13.82 \pm 1.65$ years) compared with post-menarcheal diabetes patients $(12.89 \pm 1.43$ years; $p<0.0001$; Table 1). There was a decreasing trend in the age at menarche with advancing decade of birth: the mean ages were $13.80 \pm 1.46,13.54 \pm 1.52,13.30 \pm 1.72,13.26 \pm 1.62$, $13.06 \pm 1.42$ and $12.96 \pm 1.25$ in the birth cohorts of 1944 or before, 1945-1954, 1955-1964, 1965-1974, 1975-1984 and 1985 or after $(p<0.0001$ for the trend). Mean age at menarche decreased in both the pre-menarcheal and post-menarcheal diabetes groups (Fig. 1). It should also be noted that the difference in the mean age of menarche between pre-menarcheal and post-menarcheal onset of diabetes decreased with advancing decade of birth.

There was an inverse relationship between the age at onset of diabetes and the age at menarche (Fig. 2): a younger age at onset of diabetes was associated with an older age at menarche. The age at menarche decreased linearly with increase of onset of diabetes until age 20 years.

Risk of diabetic nephropathy and severe retinopathy according to age of menarche A non-linear relationship between the age of menarche and the risk of diabetic nephropathy was found in patients with pre-menarcheal type 1 diabetes ( $p=0.004$ for non-linearity; Fig. 3a). If age at menarche was delayed by more than 2 years (corresponding to age $>15$ years at menarche), then the risk of diabetic nephropathy increased. Specifically, women with a delayed menarche ( $>$ mean age +2 years) had 2.50 times higher risk of diabetic nephropathy compared with women whose age at menarche was within \pm 2 years of the mean (OR 2.50 [95\% CI 1.54, 4.04]; $p<0.001$ ). Women with an earlier menarche ( $<$ mean age -2 years) did not have a significantly different risk of diabetic nephropathy (OR 
Table 1 Clinical characteristics of patients in the pre-menarcheal and post-menarcheal type 1 diabetes groups at the time of evaluation (1994-2011)

\begin{tabular}{|c|c|c|c|}
\hline Characteristic & Diabetes diagnosed before onset of menarche & Diabetes diagnosed after onset of menarche & $p$ value $\mathrm{fc}$ \\
\hline$n$ & 600 & 704 & \\
\hline \multicolumn{4}{|l|}{ Age (years) } \\
\hline At baseline visit & $32.5(23.9-41.5)$ & $40.2(32.3-48.1)$ & $<0.0001$ \\
\hline At onset of diabetes & $8.9(5.9-10.7)$ & $21.1(16.1-27.5)$ & $<0.0001$ \\
\hline At menarche (years) & $13.82 \pm 1.65$ & $12.89 \pm 1.43$ & $<0.0001$ \\
\hline Duration of diabetes (years) & $24.7(16.6-32.8)$ & $18.0(9.3-25.3)$ & $<0.0001$ \\
\hline BMI $\left(\mathrm{kg} / \mathrm{m}^{2}\right)$ & $24.9 \pm 3.6$ & $24.8 \pm 3.8$ & 0.48 \\
\hline WHR & $0.67 \pm 0.07$ & $0.68 \pm 0.06$ & 0.51 \\
\hline Height (cm) & $163.8 \pm 6.5$ & $165.3 \pm 6.1$ & $<0.0001$ \\
\hline $\begin{array}{l}\mathrm{HbA}_{1 \mathrm{c}}(\%) \\
\mathrm{HbA}_{1 \mathrm{c}}(\mathrm{mmol} / \mathrm{mol})\end{array}$ & $\begin{array}{l}8.5 \pm 1.4 \\
69.5 \pm 15.4\end{array}$ & $\begin{array}{l}8.2 \pm 1.4 \\
66.7 \pm 15.7\end{array}$ & 0.004 \\
\hline Total cholesterol (mmol/l) & $4.90 \pm 0.89$ & $4.88 \pm 0.84$ & 0.15 \\
\hline HDL-cholesterol (mmol/l) & $1.41 \pm 0.37$ & $1.49 \pm 0.38$ & $<.0001$ \\
\hline Triacylglycerol (mmol/l) & $0.93(0.71-1.30)$ & $0.87(0.68-1.20)$ & $<.0001$ \\
\hline $\mathrm{SBP}(\mathrm{mmHg})$ & $128.9 \pm 17.5$ & $129.5 \pm 17.1$ & $0.16^{\mathrm{b}}$ \\
\hline $\mathrm{DBP}(\mathrm{mmHg})$ & $76.8 \pm 8.9$ & $77.9 \pm 9.2$ & $0.17^{\mathrm{b}}$ \\
\hline Antihypertensive medication (\%) & 38.8 & 29.4 & $0.046^{\mathrm{b}}$ \\
\hline $\mathrm{eGFR}\left(\mathrm{ml} / \mathrm{min} / 1.73 \mathrm{~m}^{2}\right)$ & $85.7 \pm 30.0$ & $87.3 \pm 22.2$ & $0.53^{\mathrm{b}}$ \\
\hline Laser-treated retinopathy (\%) & 39.3 & 13.6 & $<0.0001$ \\
\hline Diabetic nephropathy (\%) & 19.5 & 7.1 & $<0.0001$ \\
\hline
\end{tabular}

Data are expressed as mean $( \pm \mathrm{SD})$, median (interquartile range) or percentages; $p$ value refers to ANOVA, Kruskal-Wallis test or $\chi^{2}$ test

${ }^{\text {a }}$ Adjusted for duration of diabetes when applicable

${ }^{\mathrm{b}}$ Adjusted for age and duration of diabetes

DBP, diastolic BP; eGDR, estimated GFR, calculated using the Chronic Kidney Disease Epidemiology Collaboration equation [47]

1.71 [95\% CI 0.65, 4.55]; $p=0.28)$. The effect was not much attenuated when adjusted for conventional risk factors for diabetic complications such as age at onset of diabetes, duration of diabetes, $\mathrm{HbA}_{1 \mathrm{c}}$, systolic $\mathrm{BP}, \mathrm{HDL}$, cholesterol and triacylglycerol levels, history of smoking, and BMI (OR 2.30 [95\% CI 1.27, 4.17]; Table 3). Age at menarche did not have an effect on the risk of diabetic nephropathy in women with post-menarcheal type 1 diabetes (Table 3).

Similar to diabetic nephropathy, the risk of severe diabetic retinopathy increased if the delay in menarche was more than 2 years (Fig. 3b). Delayed menarche had an increasing effect on the risk of severe retinopathy (OR 2.84 [95\% CI 1.82, 4.44]) in the univariate analysis. In the multivariable analysis, the OR was 2.34 ( $95 \%$ CI 1.36, 4.01; Table 3). When patients with diabetic nephropathy were excluded from the analysis, the effect of increasing age at menarche remained a significant predictor of severe retinopathy ( $p=0.01$ ), but the relationship was rather linear (Fig. 3c). The OR was 1.17 (95\% CI 1.04, 1.33 ) in the multivariable analysis for each increasing year of age at menarche. The comparable OR was 2.11 (95\% CI, 1.15, 3.90) for the group in which menarche was delayed by more than the mean age +2 years (Table 3 ) and the only significant predictors were duration of diabetes and age at onset of menarche.

\section{Discussion}

This is, to the best of our knowledge, the first study to analyse the association between age at menarche and risk of diabetic nephropathy and severe retinopathy in women with type 1 diabetes. Our study shows that women with pre-menarcheal type 1 diabetes and delayed menarche have an increased risk of diabetic microvascular complications.

This study confirms an earlier observation that menarche is delayed in patients with pre-menarcheal type 1 diabetes. Previous studies have reported that if the age at onset of diabetes is less than 10 years, then menarche is delayed [17, 19, 29]. We found an inverse linear relationship between the age at onset of diabetes and age at menarche, i.e. the younger the age at onset of diabetes, the older the age at menarche.

There has been debate about whether a delay in menarche still exists because diabetes management has vastly improved over the years. Recent studies, however, found that, despite intensive insulin treatment and good metabolic control, the 
Table 2 Clinical characteristics of the patients with pre-menarcheal type 1 diabetes at time of evaluation (1994-2011) according to the age at menarche

\begin{tabular}{|c|c|c|c|c|}
\hline Characteristic & $\begin{array}{l}\text { Age at } \\
\text { menarche }<\text { mean }-2 \text { years } \\
\text { (range 9-11 years) }\end{array}$ & $\begin{array}{l}\text { Age at } \\
\text { menarche }=\text { mean } \pm 2 \text { years } \\
\text { (range } 11-15 \text { years) }\end{array}$ & $\begin{array}{l}\text { Age at } \\
\text { menarche }>\text { mean }+2 \text { years } \\
\text { (range } 15-18 \text { years) }\end{array}$ & $\begin{array}{l}p \text { value for } \\
\text { difference }^{\mathrm{a}}\end{array}$ \\
\hline$n$ & 33 & 466 & 101 & \\
\hline \multicolumn{5}{|l|}{ Age (years) } \\
\hline At baseline visit & $28.2(21.0-36.3)$ & $31.5(23.5-40.5)$ & $39.0(31.7-45.8)$ & $<0.0001$ \\
\hline Onset of diabetes & $9.0(6.8-9.8)$ & $8.8(5.9-10.7)$ & $9.1(5.8-11.5)$ & 0.65 \\
\hline At menarche & $10.88 \pm 0.42$ & $13.46 \pm 1.07$ & $16.44 \pm 0.71$ & $<0.0001$ \\
\hline Duration of diabetes (years) & $18.9(14.4-27.9)$ & $23.8(15.4-31.8)$ & $30.7(22.0-38.2)$ & $<0.0001$ \\
\hline $\operatorname{BMI}\left(\mathrm{kg} / \mathrm{m}^{2}\right)$ & $25.5 \pm 3.7$ & $24.9 \pm 3.6$ & $24.6 \pm 3.5$ & 0.25 \\
\hline WHR & $0.67 \pm 0.06$ & $0.69 \pm 0.09$ & $0.67 \pm 0.07$ & 0.14 \\
\hline Height (cm) & $161.4 \pm 7.1$ & $164.6 \pm 6.3$ & $161.4 \pm 6.8$ & $<0.0001$ \\
\hline $\mathrm{HbA}_{1 \mathrm{c}}(\%)$ & $8.4 \pm 1.3$ & $8.6 \pm 1.4$ & $8.2 \pm 1.2$ & 0.10 \\
\hline $\mathrm{HbA}_{1 \mathrm{c}}(\mathrm{mmol} / \mathrm{mol})$ & $68.3 \pm 14.1$ & $70.4 \pm 15.8$ & $65.6 \pm 13.4$ & \\
\hline Total cholesterol (mmol/l) & $4.64 \pm 0.75$ & $4.91 \pm 0.89$ & $4.97 \pm 0.86$ & 0.26 \\
\hline HDL-cholesterol (mmol/l) & $1.36 \pm 0.31$ & $1.41 \pm 0.37$ & $1.40 \pm 0.38$ & 0.42 \\
\hline Triacylglycerol (mmol/l) & $0.91(0.70-1.30)$ & $0.95(0.71-1.32)$ & $0.90(0.70-1.18)$ & 0.41 \\
\hline $\mathrm{SBP}(\mathrm{mmHg})$ & $124.5 \pm 14.8$ & $128.3 \pm 17.3$ & $133.0 \pm 18.6$ & $0.88^{\mathrm{b}}$ \\
\hline $\mathrm{DBP}(\mathrm{mmHg})$ & $77.5 \pm 8.6$ & $75.9 \pm 9.4$ & $76.5 \pm 8.9$ & $0.96^{\mathrm{b}}$ \\
\hline $\begin{array}{l}\text { Antihypertensive medication } \\
(\%)\end{array}$ & 27.3 & 36.1 & 57.0 & $0.05^{\mathrm{b}}$ \\
\hline eGFR $\left(\mathrm{ml} / \mathrm{min} / 1.73 \mathrm{~m}^{2}\right)$ & $90.0 \pm 24.4$ & $88.1 \pm 29.6$ & $73.3 \pm 31.1$ & $<0.08^{\mathrm{b}}$ \\
\hline Laser-treated retinopathy $(\%)$ & 29.0 & 36.8 & 62.4 & 0.02 \\
\hline Diabetic nephropathy (\%) & 18.2 & 17.1 & 28.8 & $<0.001$ \\
\hline
\end{tabular}

Data are expressed as mean $( \pm \mathrm{SD})$, median (interquartile range) or percentages; $p$ value refers to ANOVA, Kruskal-Wallis test or $\chi^{2}$-test. There is overlap in the range of age at menarche between the groups because the age at menarche was centred to the group mean by decade

${ }^{\text {a }}$ Adjusted for duration of diabetes when applicable

${ }^{\mathrm{b}}$ Adjusted for age and duration of diabetes

DBP, diastolic BP; eGFR, estimated GFR, calculated with the Chronic Kidney Disease Epidemiology Collaboration equation [47]

menarche is still delayed for type 1 diabetes patients, but this delay does not appear to be as great as previously observed

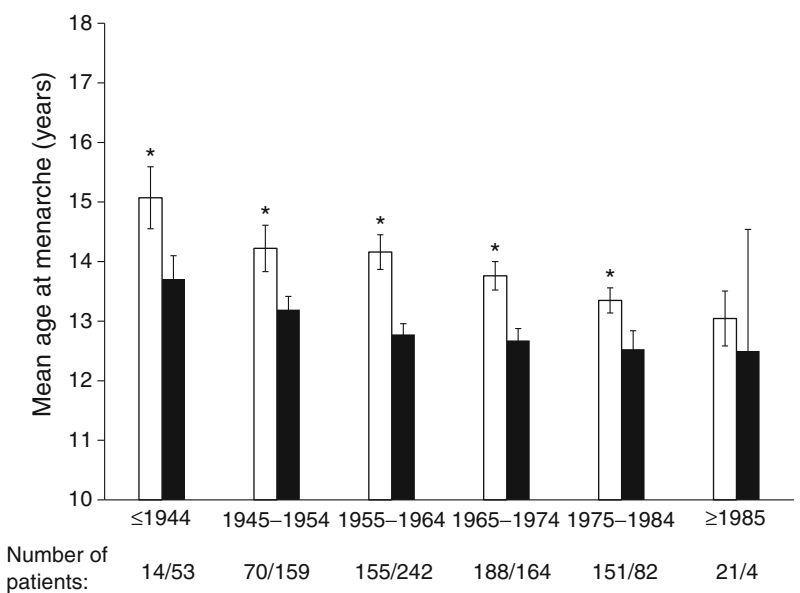

Fig. 1 Mean age at menarche in pre-menarcheal (white bars) and postmenarcheal (black bars) women with type 1 diabetes according to birth cohort. Error bars show the $95 \%$ CI. ${ }^{*} p<0.0001$ for difference. The numbers below the graph denote number of patients in the pre-menarcheal group/number of patients in the post-menarcheal group
$[10,18,30]$. The researchers concluded that factors other than glycaemic control might contribute to the menarcheal delay. Patients in our study were born over a wide time span (i.e. 1930 s to 1980s). Consistent with previous observations, a significant difference in the delay in menarche between preand post-menarcheal diabetes patients still existed in the cohort born in 1975-1984, but was diminished compared with those born in earlier decades. Owing to small sample size in the post-menarcheal group in the youngest cohort (born 1985 or later), it was not possible to make any conclusions regarding differences in age at menarche. However, comparison between patients with pre-menarcheal diabetes and a Finnish background population showed that these patients still had a slightly delayed menarche. In Finnish women without diabetes, the mean age at menarche has decreased from 14.9 years in the early 1900 s to 12.7 years in the 1970 s or later [31], as also seen worldwide [25].

One of the most important observations in the present study was that delayed menarche was associated with an increased risk of diabetic nephropathy and severe retinopathy. Unfortunately, we cannot compare our results to those of 


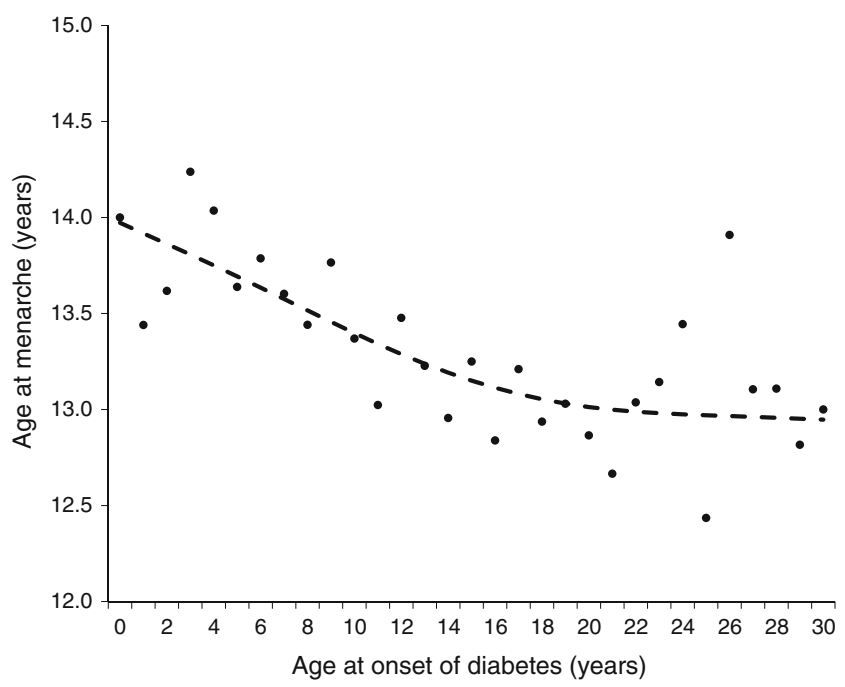

Fig. 2 Relationship between age at onset of diabetes and age at menarche in women with type 1 diabetes. Black circles denote the mean age at menarche and the dashed line (best fit) denotes the predicted age at menarche

previous studies because no comparable studies have been reported. However, delayed menarche has been associated with other diseases and conditions such as irregular menstruation and other gynaecological disturbances [17]. In the general population, menarcheal age has been associated with several other conditions, such as pre-eclampsia, decreased bone mineral density, type 2 diabetes, breast cancer and cardiovascular disease [32-34]. Puberty is known to accelerate microvascular complications. Our previous study showed that women with pubertal and prepubertal type 1 diabetes had a risk of ESRD and diabetic proliferative retinopathy that was almost equal to that of men, but that the risk diverged after puberty [1]. These data suggest that female sex as a protective factor against the development of microvascular complications may be abolished if diabetes onset is before or during puberty, thus implicating hormonal changes associated with puberty in the development of microvascular complications. Puberty is characterised by changes in the hypothalamus-pituitary-gonadal (HPG) axis, including pituitary growth hormone, insulin-like growth factor I, gonadotrophins, luteinising hormone and follicle-stimulating hormone [35]. Type 1 diabetes has been shown to cause disturbances in the HPG axis; in girls with type 1 diabetes, these disturbances are associated with

Fig. 3 Partial residual plots for GAM analyses in women with premenarcheal diabetes, showing the combined effect of linear and nonparametric contributions to deviation from the mean age at menarche (black line) on the risk of (a) diabetic nephropathy $(n=600)$, (b) severe diabetic retinopathy $(n=600$, including patients with diabetic nephropathy) and (c) severe diabetic retinopathy ( $n=495$, excluding patients with diabetic nephropathy). Shaded areas represent $95 \%$ CIs. The area above the dotted line at zero represents increased risk and the area under the dotted line represents a decreased risk a

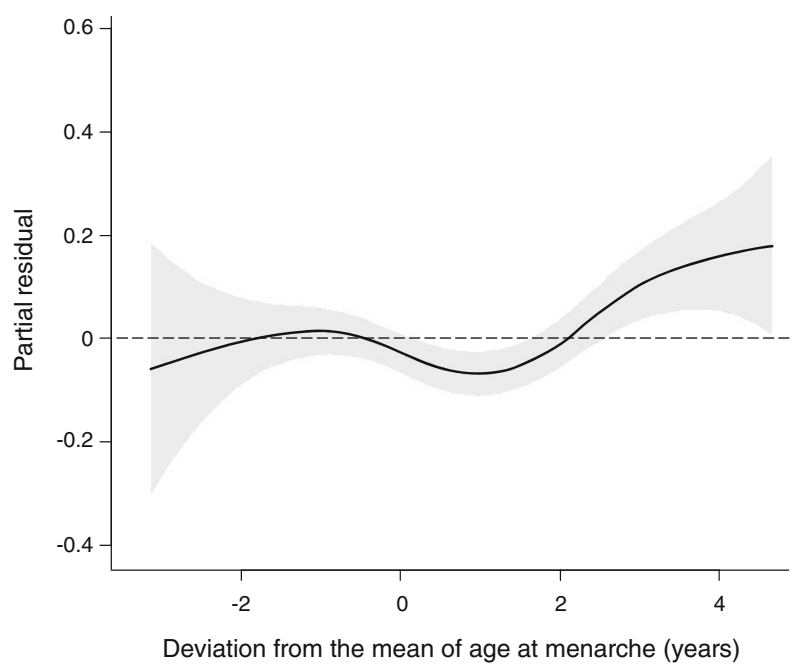

b

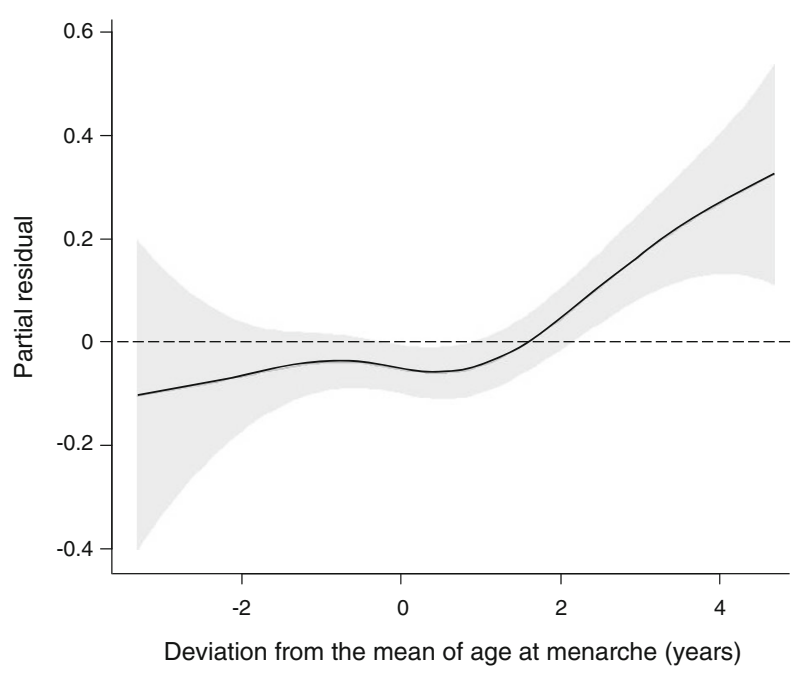

C

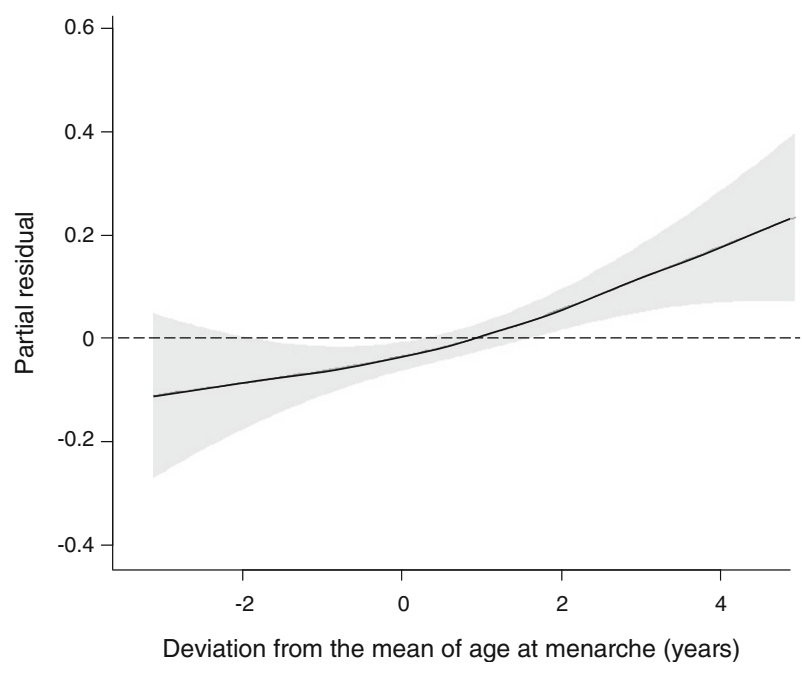


Table 3 Multivariable logistic regression analysis for the prevalence of diabetic nephropathy, laser-treated retinopathy (diabetic nephropathy included), laser-treated retinopathy (diabetic nephropathy excluded) in women with type 1 diabetes by age at menarche

\begin{tabular}{|c|c|c|c|c|c|c|}
\hline \multirow[t]{2}{*}{ Type 1 diabetes diagnosis } & \multicolumn{2}{|c|}{ Diabetic nephropathy } & \multicolumn{2}{|c|}{$\begin{array}{l}\text { Laser-treated retinopathy } \\
\text { (nephropathy included) }\end{array}$} & \multicolumn{2}{|c|}{$\begin{array}{l}\text { Laser-treated retinopathy } \\
\text { (nephropathy excluded) }\end{array}$} \\
\hline & OR $(95 \% \mathrm{CI})$ & $p$ value & OR $(95 \% \mathrm{CI})$ & $p$ value & OR $(95 \% \mathrm{CI})$ & $p$ value \\
\hline \multicolumn{7}{|l|}{ Pre-menarcheal } \\
\hline Age at menarche $<($ mean -2$)$, years & $2.09(0.76,5.72)$ & 0.15 & $0.93(0.38,2.30)$ & 0.88 & $0.69(0.22,2.19)$ & 0.53 \\
\hline Age at menarche $>($ mean +2$)$, years & $2.30(1.27,4.17)$ & 0.006 & $2.34(1.36,4.01)$ & 0.002 & $2.11(1.15,3.90)$ & 0.02 \\
\hline \multicolumn{7}{|l|}{ Post-menarcheal } \\
\hline Age at menarche $<($ mean -2$)$, years & $0.73(0.28,1.94)$ & 0.53 & $0.65(0.31,1.39)$ & 0.27 & $0.64(0.27,1.53)$ & 0.32 \\
\hline Age at menarche $>($ mean +2$)$, years & $0.30(0.02,3.65)$ & 0.34 & $0.11(0.02,1.12)$ & 0.06 & $0.23(0.03,2.04)$ & 0.19 \\
\hline
\end{tabular}

delayed ovarian maturation and sex hormone production, leading to delayed menarche [35]. Thus, there appears to be a strong link between sex hormones, delayed menarche and the development of diabetic microvascular complications associated with type 1 diabetes [36]. Further evidence for a link between hormonal status and risk of microvascular complications associated with type 1 diabetes was provided by a study showing that women with diabetic retinopathy and nephropathy undergo menopause at an earlier age [37].

Several experimental studies have demonstrated a renoprotective role for exogenous oestrogens in models of type 1 diabetic retinopathy and nephropathy [38-40]. It is possible that early onset of diabetes impairs the ovarian production of oestrogen, thus becoming permissive for the development of organ complications later in life. Indeed, in both streptozotocin-induced diabetic mice and rats, smaller ovarian preovulatory follicles and delayed oocyte maturation with increased apoptosis of ovarian follicular cells have been observed [41, 42]. Even though hyperglycaemia may cause ovarian follicle changes regardless of the age at onset, it is likely that the early onset of diabetes may not only impair oestrogen production but also permanently reduce the number of available follicles for oestrogen production later in life. Supporting this notion is a report that adult streptozotocin-induced diabetic rats exhibit decreased circulating levels of oestrogen and an imbalance in the expression of renal oestrogen receptors [43].

In the present study, patients with a delayed menarche had the best glycaemic control. In addition, it should be noted that, while our data indicate that women with delayed menarche had the best $\mathrm{HbA}_{1 \mathrm{c}}$ values at the baseline visit, these values represent glycaemic control several years, or even decades, after puberty. The reason for delayed menarche may be poor metabolic control during the second decade of life, which is a critical time for the development of chronic complications. Menarche is one of the final events of puberty, and delayed puberty and menarche may indicate diminished oestrogen levels during puberty. Therefore, worse metabolic control may leads to mild hypogonadism, delayed puberty and delayed menarche.

It is widely accepted that the substantial variation in age at menarche among individuals may be due to genetic factors [44]. A recent large genome-wide association study detected 106 genomic loci involving in pubertal timing and age at menarche [45]. While it is unlikely that age at menarche per se is a causal factor for diabetic nephropathy or retinopathy, it may be a marker of an underlying process that confers an increased risk. Recently, our study group belonging to the GENIE ('GEnetics of Nephropathy an International Effort') consortium identified the first sex-specific genetic variant to be strongly associated with ESRD in type 1 diabetes in women but not in men [46]. Interestingly, the variant is located between the SP 3 and $C D C A 7$ genes, and SP 3 encodes a transcription factor that directly interacts with oestrogen receptor alpha $(\mathrm{ER}-\alpha)$.

Finally, some limitations need to be considered. As with any study that uses self-reported data on questionnaires, recall bias may exist. Another limitation is that the $\mathrm{HbA}_{1 \mathrm{c}}$ values used in the present study were measured at the baseline visit, i.e. years or even decades after menarche. It is therefore not possible to rule out the possibility that patients with delayed menarche had worse glycaemic control during the pubertal period.

\section{Conclusions}

Our study shows that age at onset of type 1 diabetes contributes to the age at menarche and, in turn, to the risk of diabetic nephropathy and severe retinopathy later in life. Specifically, the relation between age at menarche and the risk of diabetic nephropathy and severe retinopathy was J-shaped, i.e. delayed menarche was associated with an increased risk of diabetic nephropathy and severe retinopathy but early menarche was not. Delayed menarche may thus be used as a new tool to 
identify women at risk of diabetic microvascular complications. It is, however, possible that in the future a shorter delay than 2 years could be used as a sign of delayed menarche and thus as a marker of increased risk of diabetic microvascular complications.

Acknowledgements The authors would like to acknowledge all of the physicians and nurses at each of the FinnDiane centres that participated in patient recruitment and characterisation (see the electronic supplementary material [ESM1].

Funding This research was funded by grants from the Folkhälsan Research Foundation, the Academy of Finland (134379), the Wilhelm and Else Stockmann Foundation, the Liv och Hälsa Foundation and the Novo Nordisk Foundation. The funding bodies were not involved in the design or conduct of the study.

Duality of interest statement P-HG has received lecture honorariums from AbbVie, Boehringer Ingelheim, Cebix, Eli Lilly and Company, Genzyme, Novartis, Novo Nordisk, MSD and Medscape, and research grants from Eli Lilly and Company and Roche. P-HG is also an advisory board member of Boehringer Ingelheim, Eli Lilly and Company and Novartis. All other authors declare that there is no duality of interest associated with their contribution to this manuscript

Contribution statement VH was responsible for study and questionnaire design, statistical analyses and first draft of the manuscript. CM-B contributed to the study and questionnaire design and to the writing and critical revision of the manuscript. CF contributed to the questionnaire design, data acquisition and critical revision of the manuscript. P-HG was the principal investigator of the study and participated in interpretation of the results and critical revision of the manuscript. All authors contributed to the final version. P-HG is responsible for the integrity of the work as a whole.

\section{References}

1. Harjutsalo V, Maric C, Forsblom C et al (2011) Sex-related differences in the long-term risk of microvascular complications by age at onset of type 1 diabetes. Diabetologia 54:1992-1999

2. Finne P, Reunanen A, Stenman S, Groop PH, Gronhagen-Riska C (2005) Incidence of end-stage renal disease in patients with type 1 diabetes. JAMA 294:1782-1787

3. Hietala K, Harjutsalo V, Forsblom C, Summanen P, Groop PH (2010) Age at onset and the risk of proliferative retinopathy in type 1 diabetes. Diabetes Care 33:1315-1319

4. Krolewski AS, Warram JH, Christlieb AR, Busick EJ, Kahn CR (1985) The changing natural history of nephropathy in type I diabetes. Am J Med 78:785-794

5. Andersen AR, Christiansen JS, Andersen JK, Kreiner S, Deckert T (1983) Diabetic nephropathy in type 1 (insulin-dependent) diabetes: an epidemiological study. Diabetologia 25:496-501

6. Kostraba JN, Dorman JS, Orchard TJ et al (1989) Contribution of diabetes duration before puberty to development of microvascular complications in IDDM subjects. Diabetes Care 12:686-693

7. Lawson ML, Sochett EB, Chait PG, Balfe JW, Daneman D (1996) Effect of puberty on markers of glomerular hypertrophy and hypertension in IDDM. Diabetes 45:51-55

8. Svensson M, Nystrom L, Schon S, Dahlquist G (2006) Age at onset of childhood-onset type 1 diabetes and the development of end- stage renal disease: a nationwide population-based study. Diabetes Care 29:538-542

9. Schultz CJ, Konopelska-Bahu T, Dalton RN et al (1999) Microalbuminuria prevalence varies with age, sex, and puberty in children with type 1 diabetes followed from diagnosis in a longitudinal study. Oxford Regional Prospective Study Group. Diabetes Care 22:495-502

10. Schweiger BM, Snell-Bergeon JK, Roman R, McFann K, Klingensmith GJ (2011) Menarche delay and menstrual irregularities persist in adolescents with type 1 diabetes. Reprod Biol Endocrinol: 9:61

11. Rudberg S, Ullman E, Dahlquist G (1993) Relationship between early metabolic control and the development of microalbuminuriaa longitudinal study in children with type 1 (insulin-dependent) diabetes mellitus. Diabetologia 36:1309-1314

12. Codner E, Cassorla F (2009) Puberty and ovarian function in girls with type 1 diabetes mellitus. Horm Res 71:12-21

13. Mauer M, Drummond K (2002) The early natural history of nephropathy in type 1 diabetes: I. Study design and baseline characteristics of the study participants. Diabetes 51:1572-1579

14. Drummond KN, Kramer MS, Suissa S et al (2003) Effects of duration and age at onset of type 1 diabetes on preclinical manifestations of nephropathy. Diabetes 52:1818-1824

15. Janner M, Knill SE, Diem P, Zuppinger KA, Mullis PE (1994) Persistent microalbuminuria in adolescents with type I (insulindependent) diabetes mellitus is associated to early rather than late puberty. Results of a prospective longitudinal study. Eur J Pediatr 153:403-408

16. Codner E (2008) Estrogen and type 1 diabetes mellitus. Pediatr Endocrinol Rev 6:228-234

17. Deltsidou A (2010) Age at menarche and menstrual irregularities of adolescents with type 1 diabetes. J Pediatr Adolesc Gynecol 23:162-167

18. Lombardo F, Salzano G, Crisafulli G et al (2009) Menarcheal timing in intensively treated girls with type 1 diabetes mellitus. Nutr Metab Cardiovasc Dis: NMCD 19:35-38

19. Kjaer K, Hagen C, Sando SH, Eshoj O (1992) Epidemiology of menarche and menstrual disturbances in an unselected group of women with insulin-dependent diabetes mellitus compared to controls. J Clin Endocrinol Metab 75:524-529

20. Zhang H, Xia W, Yu Q et al (2008) Increasing incidence of type 1 diabetes in children aged 0-14 years in Harbin, China (1990-2000). Prim Care Diabetes 2:121-126

21. Soltesz G, Patterson CC, Dahlquist G (2007) Worldwide childhood type 1 diabetes incidence-what can we learn from epidemiology? Pediatr Diabetes 8(Suppl 6):6-14

22. Lammi N, Taskinen O, Moltchanova E et al (2007) A high incidence of type 1 diabetes and an alarming increase in the incidence of type 2 diabetes among young adults in Finland between 1992 and 1996. Diabetologia 50:1393-1400

23. Ostman J, Lonnberg G, Arnqvist HJ et al (2008) Gender differences and temporal variation in the incidence of type 1 diabetes: results of 8012 cases in the nationwide Diabetes Incidence Study in Sweden 1983-2002. J Intern Med 263:386-394

24. Thorn LM, Forsblom C, Fagerudd J et al (2005) Metabolic syndrome in type 1 diabetes: association with diabetic nephropathy and glycemic control (the FinnDiane study). Diabetes Care 28:2019-2024

25. Codner E, Soto N, Merino PM (2012) Contraception and pregnancy in adolescents with type 1 diabetes: a review. Pediatr Diabetes 13:108-123

26. Hastie TJ, Tibshirani RJ (1990) Generalized additive models. Chapman \& Hall/CRC, London

27. Wood SN (2006) Generalized additive models: an introduction with R. Chapman \& Hall/CRC, London 
28. Wood S (2015) Mixed GAM computation vehicle with GCV/AIC/ REML smoothness estimation. Available from https://cran.rproject.org/web/packages/mgcv/mgcv.pdf. Accessed 9 Nov 2015

29. Yeshaya A, Orvieto R, Dicker D, Karp M, Ben-Rafael Z (1995) Menstrual characteristics of women suffering from insulin-dependent diabetes mellitus. Int J Fertil Menopausal Stud 40:269-273

30. Picardi A, Cipponeri E, Bizzarri C, Fallucca S, Guglielmi C, Pozzilli P (2008) Menarche in type 1 diabetes is still delayed despite good metabolic control. Fertil Steril 90:1875-1877

31. Koponen P, Luoto R (2004) Reproductive health in Finland. Health 2000 Survey. Publications of National Public Health Institute, Helsinki [article in Finnish]

32. Abetew DF, Enquobahrie DA, Dishi M, Rudra CB, Miller RS, Williams MA (2011) Age at menarche, menstrual characteristics, and risk of preeclampsia. ISRN Obstet Gynecol 2011:472083

33. Janghorbani M, Mansourian M, Hosseini E (2014) Systematic review and meta-analysis of age at menarche and risk of type 2 diabetes. Acta Diabetol 51:519-528

34. Prentice P, Viner RM (2013) Pubertal timing and adult obesity and cardiometabolic risk in women and men: a systematic review and meta-analysis. Int J Obes 37:1036-1043

35. Codner E, Merino PM, Tena-Sempere M (2012) Female reproduction and type 1 diabetes: from mechanisms to clinical findings. Hum Reprod Update 18:568-585

36. Lane PH (2002) Diabetic kidney disease: impact of puberty. Am J Physiol Renal Physiol 283:F589-F600

37. Sjoberg L, Pitkaniemi J, Harjutsalo V et al (2011) Menopause in women with type 1 diabetes. Menopause 18:158-163

38. Mankhey RW, Bhatti F, Maric C (2005) 17 $\beta$-Estradiol replacement improves renal function and pathology associated with diabetic nephropathy. Am J Physiol Renal Physiol 288:F399-F405
39. Keck M, Romero-Aleshire MJ, Cai Q, Hoyer PB, Brooks HL (2007) Hormonal status affects the progression of STZ-induced diabetes and diabetic renal damage in the VCD mouse model of menopause. Am J Physiol Renal Physiol 293:F193-F199

40. Kaja S, Yang SH, Wei J et al (2003) Estrogen protects the inner retina from apoptosis and ischemia-induced loss of Vesl-1L/Homer $1 \mathrm{c}$ immunoreactive synaptic connections. Invest Ophthalmol Vis Sci 44:3155-3162

41. Chabrolle C, Jeanpierre E, Tosca L, Rame C, Dupont J (2008) Effects of high levels of glucose on the steroidogenesis and the expression of adiponectin receptors in rat ovarian cells. Reprod Biol Endocrinol 6:11

42. Bestetti GE, Junker U, Locatelli V, Rossi GL (1987) Continuous subtherapeutic insulin counteracts hypothalamopituitary-gonadal alterations in diabetic rats. Diabetes 36:1315-1319

43. Wells CC, Riazi S, Mankhey RW, Bhatti F, Ecelbarger C, Maric C (2005) Diabetic nephropathy is associated with decreased circulating estradiol levels and imbalance in the expression of renal estrogen receptors. Gend Med 2:227-237

44. Towne B, Czerwinski SA, Demerath EW, Blangero J, Roche AF, Siervogel RM (2005) Heritability of age at menarche in girls from the Fels Longitudinal Study. Am J Phys Anthropol 128:210-219

45. Perry JR, Day F, Elks CE et al (2014) Parent-of-origin-specific allelic associations among 106 genomic loci for age at menarche. Nature 514:92-97

46. Sandholm N, McKnight AJ, Salem RM et al (2013) Chromosome $2 \mathrm{q} 31.1$ associates with ESRD in women with type 1 diabetes. J Am Soc Nephrol 24:1537-1543

47. Levey AS, Stevens LA, Schmid CH et al (2009) CKD-EPI (Chronic Kidney Disease Epidemiology Collaboration). A new equation to estimate glomerular filtration rate. Ann Intern Med 150:604-612 